\title{
Systematic review of incidence, risk factors, prevention and treatment of post-laryngectomy hypoparathyroidism
}

\author{
Ovie Edafe $^{1}\left(\mathbb{D} \cdot\right.$ Luke M. Sandler $^{2} \cdot$ Nigel Beasley $^{1} \cdot$ Sabapathy P. Balasubramanian $^{3,4}$
}

Received: 24 April 2020 / Accepted: 14 July 2020 / Published online: 22 July 2020

(c) The Author(s) 2020

\begin{abstract}
Purpose Post-laryngectomy hypoparathyroidism is associated with significant short- and long-term morbidities. This systematic review aimed to determine incidence, risk factors, prevention and treatment of post-laryngectomy hypoparathyroidism. Methods Medline, EMBASE and the Cochrane library were searched for relevant articles on hypocalcaemia and/or hypoparathyroidism after laryngectomy or pharyngectomy. Two authors independently screened titles and abstracts from the search. Data from individual studies were collated and presented (without meta-analysis). Quality assessment of included studies was undertaken. The review protocol was registered in the PROSPERO database (CRD42019133879).

Results Twenty-three observational studies were included. The rates of transient and long-term hypoparathyroidism following laryngectomy with concomitant hemi- or total thyroidectomy ranged from 5.6 to $57.1 \%$ ( $n=13$ studies) and 0 to $12.8 \%$ $(n=5$ studies), respectively. Higher transient (62.1-100\%) and long-term (12.5-91.6\%) rates were reported in patients who had concomitant oesophagectomy and total thyroidectomy ( $n=4$ studies). Other risk factors included bilateral selective lateral neck dissection, salvage laryngectomy and total pharyngectomy. There is a lack of data on prevention and management. Conclusion Hypoparathyroidism occurs in a significant number of patients after laryngectomy. Patients who underwent laryngectomy with concomitant hemithyroidectomy may still develop hypoparathyroidism. Research on prevention and treatment is lacking and needs to be encouraged.
\end{abstract}

Keywords Laryngectomy $\cdot$ Pharyngectomy $\cdot$ Oesophagectomy $\cdot$ Hypocalcaemia $\cdot$ Hypoparathyroidism

\section{Introduction}

Post-surgical hypocalcaemia or hypoparathyroidism (PoSH) is a well-documented complication of central compartment neck surgery, particularly thyroidectomy [1]. This is due to direct damage, devascularisation or inadvertent excision of adjacent parathyroid glands.

The reported rates (i.q.r.) of transient and long-term hypocalcaemia following bilateral thyroid surgery were

Ovie Edafe

ovieedafe@ hotmail.co.uk

1 Department of ENT, Sheffield Teaching Hospitals Foundation Trust, Sheffield, UK

2 Emergency Department, Croydon University Hospital, London, UK

3 Oncology and Metabolism, University of Sheffield, Sheffield, UK

4 Department of General Surgery, Sheffield Teaching Hospitals Foundation Trust, Sheffield, UK
27\% (19-38) and 1\% (0-3) [1]. The estimated prevalence of PoSH in Europe and United States of America was 22 per 100,000 and 23 per 100,000, respectively [2]. Risk factors for PoSH following thyroid surgery include inadvertent parathyroid gland excision, identification of fewer than two parathyroid glands, heavier thyroid gland, reoperation for bleeding, surgery for recurrent goitre, retrosternal goitre, Graves' disease and central neck dissection [1].

PoSH has significant short- and long-term morbidity. Acute hypocalcaemia may present with paraesthesia, neuromuscular irritability, cardiac arrhythmias, and seizures [3]. Clinical features of long-term hypocalcaemia and/or hypoparathyroidism include renal impairment, seizures, neuropsychiatric diseases and infections $[4,5]$.

A number of preventative measures have been evaluated in thyroid surgery, including the use of routine postoperative supplementation, autotransplantation and haemostatic techniques.

Patients undergoing laryngectomy usually have concomitant unilateral or bilateral thyroidectomy and, therefore, are 
at risk of developing hypocalcaemia [6]. This is mostly secondary to hypoparathyroidism (risk increased by extent of primary surgery) as other mechanisms of hypocalcaemia such as hungry bone syndrome seen in thyroid surgery do not play a significant role in this scenario. The incidence of transient and long-term PoSH following laryngectomy is not well documented. In addition, risk factors, preventative measures and treatment strategies are not well described.

The aim of this systematic review was to evaluate reported incidence, risk factors, preventative measures and treatment strategies of post-laryngectomy hypocalcaemia or hypoparathyroidism.

\section{Methods}

Medline, EMBASE and Cochrane library databases were searched from the inception of the databases to January 2020 using the key words: (laryngectomy OR pharyngectomy) AND (hypocalc* OR hypoparathyroidism OR low calcium) AND (incidence OR prediction OR risk factors OR preventative measure OR prevention OR treatment OR management OR protocol). All human observational and interventional studies reporting on incidence, risk factors, predictors or management of $\mathrm{PoSH}$ following laryngectomy were eligible for inclusion. We excluded case reports, reviews, letters, commentaries, animal studies, and articles not available in English language. The bibliography of included studies was searched to identify additional articles for inclusion.

The main outcomes were the proportion of patients who developed transient and long-term PoSH. The rates of transient and long-term PoSH were reported as defined by individual studies.

Two researchers independently screened titles and abstracts from initial search. Relevant full texts were retrieved and further evaluated for eligibility against the criteria listed above. Data were extracted from included studies using a standardised data collection tool by one reviewer and checked for accuracy by another reviewer. Extracted data included details of study characteristics, population studied, definitions of outcomes and data on any risk factors, preventative methods and management that may be described in the study.

The methodological quality of included studies was assessed using a tool described by Murad et al. [7]. This tool gives a total score of six: based on selection, ascertainment of exposure and outcome, confounding factors, follow-up, and clarity of the report. The quality of articles was assessed as low, medium and high for scores of $0-2,3-4$, and 5-6, respectively.

Quantitative synthesis was not done due to heterogeneity in the extent of surgery and definitions of PoSH used in included studies. Odds ratio (OR) was provided only if reported in the individual studies.

This review was registered with PROSPERO database (CRD42019133879).

\section{Results}

Twenty-three observational studies published between 1968 and 2019 on 1416 patients were included in this review [6, 8-29]. The studies were from Europe $(n=11)$, North America $(n=4)$, Africa $(n=4)$, Asia $(n=3)$, and South America $(n=1)$. Of these, $23(100 \%)$ reported on rates of PoSH, 7 (30.4\%) on risk factors, one $(4.3 \%)$ on prevention and none $(0 \%)$ on the effectiveness of treatment strategies. There were no interventional studies.

Figure 1 shows the process of study selection. "Wrong population" represented articles that did not evaluate PoSH following laryngectomy, and "inadequate data" represented articles where the rates of PoSH could not be calculated based on the information provided. The median (i.q.r.) number of patients in the 23 studies was 47 (25-81). Fourteen studies (60.9\%) were focused on PoSH, while 9/23 (39.1\%) reported on PoSH as a secondary outcome. The median (i.q.r) quality assessment score for the included study was 3 (3.0-3.5). Table 1 shows the quality assessment for each study.

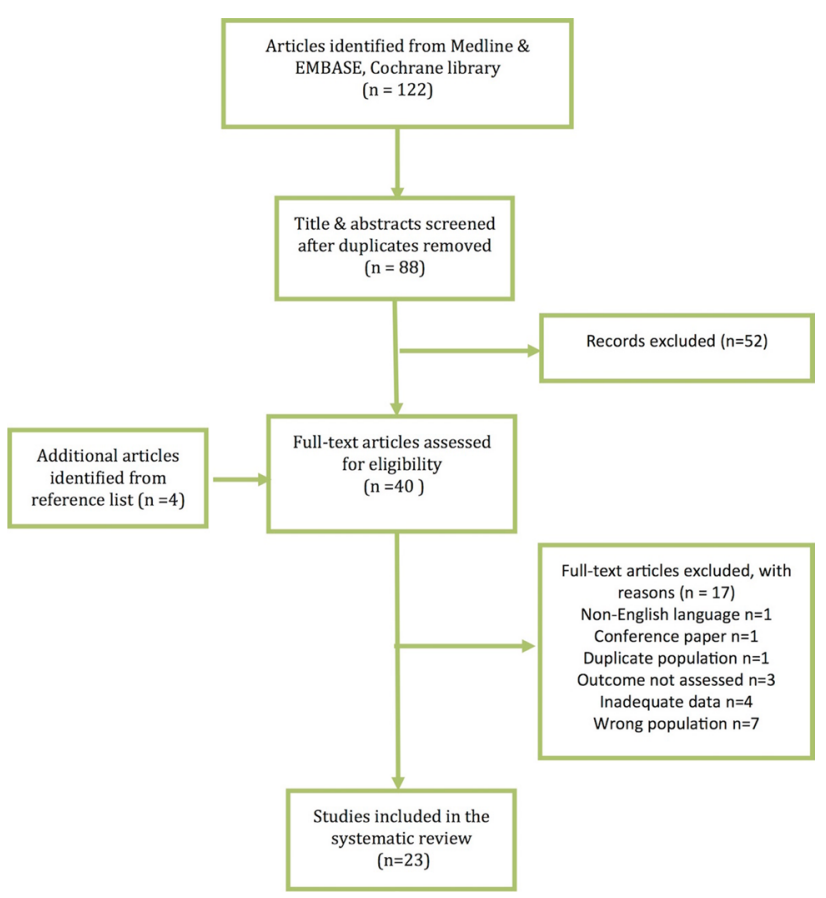

Fig. 1 PRISMA flow diagram showing steps of inclusion and exclusion of articles 
Table 1 Quality assessment of included studies

\begin{tabular}{llllllll}
\hline Studies & Q1 & Q2 & Q3 & Q4 & Q5 & Q6 & Total \\
\hline Farlow et al. [29] & 0 & 1 & 1 & 0 & 1 & 0 & 3 \\
Saito et al. [28] & 0 & 1 & 0 & 0 & 1 & 0 & 2 \\
Panda et al. [27] & 0 & 1 & 1 & 0 & 1 & 0 & 3 \\
Harris et al. [6] & 1 & 1 & 1 & 0 & 1 & 1 & 5 \\
Negm et al. [17] & 0 & 1 & 1 & 0 & 1 & 0 & 3 \\
Okano et al. [18] & 0 & 1 & 1 & 0 & 1 & 0 & 3 \\
Shenson et al. [20] & 1 & 1 & 0 & 0 & 1 & 0 & 3 \\
Marion et al. [15] & 0 & 1 & 0 & 0 & 1 & 0 & 2 \\
Abd Elmaksoud et al. [8] & 0 & 1 & 1 & 1 & 1 & 1 & 5 \\
Basheeth et al. [9] & 1 & 1 & 1 & 0 & 1 & 1 & 5 \\
Gurbuz et al. [11] & 0 & 1 & 0 & 0 & 1 & 0 & 2 \\
Oosthuizen et al. [19] & 1 & 1 & 0 & 0 & 1 & 0 & 3 \\
Leong et al. [13] & 1 & 1 & 0 & 0 & 1 & 0 & 3 \\
Lo Galbo et al. [14] & 0 & 1 & 1 & 0 & 1 & 0 & 3 \\
Martins et al. [25] & 1 & 1 & 0 & 0 & 1 & 0 & 3 \\
Clark et al. [10] & 1 & 1 & 1 & 0 & 1 & 0 & 4 \\
Thorp et al. [23] & 0 & 1 & 1 & 0 & 1 & 0 & 3 \\
Mortimore et al. [16] & 0 & 1 & 1 & 0 & 1 & 0 & 3 \\
Smith et al. [21] & 0 & 1 & 0 & 0 & 1 & 0 & 2 \\
Krespi et al. [22] & 0 & 1 & 0 & 0 & 1 & 0 & 2 \\
Harrison et al. [12] & 1 & 1 & 1 & 0 & 0 & 0 & 3 \\
Buchanan et al. [26] & 1 & 1 & 1 & 0 & 1 & 1 & 5 \\
Osborn et al. [24] & 1 & 1 & 1 & 0 & 1 & 1 & 5 \\
Median total & & & & & & & 3 \\
\hline & & 1 & 0 & 0 & 0 \\
\hline
\end{tabular}

Q1 selection methods, $Q 2$ ascertainment of exposure, $Q 3$ assessment of outcome, $Q 4$ alternative causes evaluated, $Q 5$ adequate follow-up, $Q 6$ report described in sufficient details
Table 2 shows the characteristics of included studies, definition for PoSH and proportion of patients who had transient and/or long-term PoSH in individual studies. Table 3 gives the reported range of hypocalcaemia stratified by extent of surgery. In patients having total laryngectomy with concomitant hemi/total thyroidectomy, the reported rates of transient and long-term PoSH ranged from 5.6 to $59.3 \%$ and 0 to $12.8 \%$, respectively.

\section{Extent of primary surgery}

Harris et al. compared the rates of transient and long-term PoSH in laryngectomy patients who had total thyroidectomy versus hemithyroidectomy or thyroid preservation [6]. Total thyroidectomy significantly increased the rates of transient (OR 15.5, 95\% CI 2.2, 181.9) and long term (OR 22.7, 95\% CI 1.9, 376.5) PoSH. Interestingly, Basheeth et al. found no statistically significant difference in the rates of transient PoSH between concomitant total thyroidectomy (57\%) versus lobectomy/isthmusectomy (35.9\%) in 60 patients following laryngectomy [9].

Clark et al. explored the relationship between pharyngectomy and transient $\mathrm{PoSH}$ in patients undergoing laryngopharyngectomy and flap reconstruction. In their multivariable analyses, circumferential pharyngectomy significantly increased the rates of transient PoSH compared to partial pharyngectomy $(P=0.017$; OR 3.91). In addition, laparotomy to do a free jejunum flap or gastric transposition (compared to fasciocutaneous flaps) was associated with increased risk of transient PoSH [10]. Basheeth et al. found no association between total/subtotal pharyngectomy and transient $\mathrm{PoSH}$ in a population undergoing laryngectomy for laryngeal or hypopharyngeal cancer [9].

In the context of laryngo-pharyngo-oesophagectomy for cervical oesophageal cancer, Saito et al. found total oesophagectomy (compared to partial) increased the risk of long-term PoSH [28].

Furthermore, previous tracheostomy prior to laryngectomy was not found to be associated with transient PoSH [9] .

\section{Extent of nodal surgery}

Basheeth et al. also evaluated the association between selective lateral neck dissection and transient POSH in 60 patients undergoing laryngectomy. In their multivariable analysis, 
Table 2 Characteristics of included studies




Table 2 (continued)

\begin{tabular}{|c|c|c|c|c|c|}
\hline Studies & Population & $\begin{array}{l}\text { Extent of thyroidec- } \\
\text { tomy }\end{array}$ & $\begin{array}{l}\text { Definition of transient } \\
\text { hypocalcaemia }\end{array}$ & $\begin{array}{l}\text { Definition of long-term } \\
\text { hypocalcaemia }\end{array}$ & $\begin{array}{l}\text { Overall rates of } \\
\text { transient }(\mathrm{T}) \text { and long } \\
\text { term }(\mathrm{L})\end{array}$ \\
\hline $\begin{array}{l}\text { Martins et al. [25] } \\
\text { Brazil } \\
n=40\end{array}$ & Total LPO & $\begin{array}{l}\text { None: } 3 \\
\text { Partial: } 25 \\
\text { Total: } 12\end{array}$ & $\begin{array}{l}\text { Clinical signs and } \\
\text { symptoms of hypoc- } \\
\text { alcaemia }\end{array}$ & Not applicable & $\mathrm{L}: 22 / 40(55 \%)$ \\
\hline $\begin{array}{l}\text { Thorp et al. [23] } \\
\text { South Africa } \\
n=20\end{array}$ & $\mathrm{TL} \pm$ partial $\mathrm{P}$ & Hemi: 20 & $\mathrm{cCa}<2.10 \mathrm{mmol} / \mathrm{L}$ & Not applicable & $\mathrm{T}: 7 / 20(35 \%)$ \\
\hline $\begin{array}{l}\text { Mortimore et al. } \\
{[16]} \\
\text { South Africa } \\
n=30\end{array}$ & $\begin{array}{l}\text { TL: } 19 \\
\text { TL + partial P: } 11\end{array}$ & Hemi: 30 & Not reported & Not applicable & $\mathrm{T}: 2 / 30(6.7 \%)$ \\
\hline $\begin{array}{l}\text { Smith et al. [21] } \\
\text { UK }\end{array}$ & $\begin{array}{l}\text { TL: } 20 \\
\text { Total LP: } 7\end{array}$ & $\begin{array}{l}\text { Hemi: } 19 \\
\text { Total: } 8\end{array}$ & Not reported & Not applicable & T: 16/27 (59.3\%) \\
\hline $\begin{array}{l}\text { Krespi et al. [22] } \\
\text { USA } \\
n=47\end{array}$ & $\begin{array}{l}\text { TL: } 17 \\
\text { Total LPO: } 30\end{array}$ & Not reported & $\begin{array}{l}\text { Need for postoperative } \\
\text { supplementation }\end{array}$ & Not applicable & T: 24/47 (51.1\%) \\
\hline $\begin{array}{l}\text { Harrison et al. [12] } \\
\text { UK } \\
n=58\end{array}$ & Total LPO & Total: 58 & $\mathrm{cCa}<2 \mathrm{mmol} / \mathrm{L}$ & Not applicable & T: 36/58 (62.1\%) \\
\hline $\begin{array}{l}\text { Buchanan et al. } \\
\text { [26] } \\
\text { UK } \\
n=6\end{array}$ & Total LPO & Total: 6 & $\mathrm{cCa}<2.00 \mathrm{mmol} / \mathrm{L}$ & Not applicable & $\mathrm{T}: 5$ patients \\
\hline $\begin{array}{l}\text { Osborn et al. [24] } \\
\text { UK } \\
n=15\end{array}$ & $\begin{array}{l}\text { TL: } 13 \\
\text { TL + partial P: } 2\end{array}$ & $\begin{array}{l}\text { Isthmus: } 9 \\
\text { Hemi/isthmus: } 6\end{array}$ & $\begin{array}{l}\text { Serum cal- } \\
\text { cium }<8.6 \mathrm{mg} / \mathrm{dL}\end{array}$ & Not applicable & T: 7/15 (46.7\%) \\
\hline
\end{tabular}

$T L$ total laryngectomy, $P$ pharyngectomy, $L P$ laryngopharyngectomy, $L P O$ laryngopharyngo-oesophagectomy, $c C a$ corrected calcium, $P T H$ parathyroid hormone

Table 3 Rates of transient and long-term hypocalcaemia by surgical extent

\begin{tabular}{lcc}
\hline Extent of surgery $^{\mathrm{a}}$ & $\begin{array}{l}\text { Transient } \\
(\%)\end{array}$ & $\begin{array}{l}\text { Long term } \\
(\%)\end{array}$ \\
\hline Laryngectomy with hemithyroidectomy or total thyroidectomy & $5.6-59.3[6,9,10,13,14,16,17,19-21$, & $0-12.8[6,11,14,27,29]$ \\
& $23,24,29]$ & $0-7[6,14,27]$ \\
Laryngectomy and hemithyroidectomy & $6.7-47.3[6,9,14,16,17,21,23]$ & $60-68.8[6,27]$ \\
Laryngectomy and total thyroidectomy & $43-87.5[6,9,21]$ & $44[25]$ \\
Laryngectomy with oesophagectomy and hemithyroidectomy & $2.6-70.6[18,25,28]$ & $12.5-91.6[8,25]$ \\
Laryngectomy with oesophagectomy and total thyroidectomy & $62.1-100[8,12,18,25]$ & \\
\hline
\end{tabular}

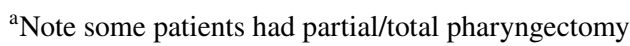

they found bilateral selective neck dissection increased the risk of transient PoSH (OR 4.56, 95\% CI 1.26, 15.80) [9].

Two studies evaluated paratracheal lymph node dissection and transient PoSH [14, 29]. Lo Galbo et al. evaluated complication associated with paratracheal neck dissection in 137 patients who had laryngectomy with or without hemithyroidectomy [14]. They excluded patients who had total thyroidectomy as part of the primary operation. They found bilateral paratracheal lymph node dissection did not significantly increase the rates of transient PoSH compared to unilateral paratracheal lymph node dissection (50\% versus $50 \%)$. Farlow et al. examined the effects of paratracheal neck 
dissection in 210 patients who underwent salvage laryngectomy [29]. They found no significant difference in rates of transient PoSH in patients who had bilateral PTLN, unilateral PTLN, and no PTLN (17\% versus 6\% versus $8 \%$, respectively). In addition, the rates of long-term PoSH were not significantly different between the groups.

\section{Primary versus salvage surgery}

Three studies evaluated the association between salvage laryngectomy and transient PoSH [9, 10, 28]. Basheeth et al. found salvage laryngectomy increased the risk of transient $\mathrm{PoSH}$ in their multivariable analysis (OR 4.83, 95\% CI 1.17-20.00). Clark et al. examined morbidity after flap reconstruction in tumour involving the hypopharynx. In their univariable analysis, they found no statistical significant difference in the rates of hypocalcaemia between primary $(51 \%)$ and salvage (40\%) surgery [10]. Another study of 17 patients with cervical oesophageal tumour found no association between chemoradiotherapy treatment and hypoparathyroidism following surgery [28].

\section{Tumour stage}

One study evaluated the association between tumour stage and hypoparathyroidism in 60 patients undergoing laryngectomy [9]. They found pT4 stage tumour stage was not associated with transient PoSH.

\section{Postoperative radiotherapy}

In a study of 17 patients, Negm et al. found no association between postoperative radiotherapy and transient PoSH. They excluded patients who had total thyroidectomy as part of laryngectomy [17].

\section{Prevention}

The effect of parathyroid gland autotransplantation on hypocalcaemia was studied in 24 patients who underwent laryngectomy with total thyroidectomy [8]. The parathyroid glands were excised, stored in ice, and following histological confirmation and exclusion of malignancy were autotransplanted onto the anterior forearm muscles. The patients received routine calcitriol (25-50 ug) and calcium carbonate (2-3 g). All developed transient hypocalcaemia within 5 days postoperatively. At 6 months, three patients (12.5\%) were on calcium and vitamin D supplements.

\section{Treatment}

There was no study evaluating the effectiveness of treatment strategies or management protocol on PoSH following laryngectomy.

\section{Discussion}

To our knowledge, this is the first systematic review on hypocalcaemia or hypoparathyroidism following laryngectomy.

This review aimed to ascertain reported rates of hypocalcaemia or hypoparathyroidism following laryngectomy and to identify risk factors, preventive measures and treatment strategies described in the literature. Contrary to thyroid surgery, patients who underwent laryngectomy with hemithyroidectomy may still develop transient and long-term hypocalcaemia regardless of the preservation of the contralateral thyroid lobe. However, the reported rates of transient and long-term hypocalcaemia were higher in patients who had total thyroidectomy (Table 3). Transient hypocalcaemia can occur in up to $100 \%$ of patients who had total laryngectomy with concomitant oesophagectomy and total thyroidectomy $[8,18,25]$; long-term hypocalcaemia is also higher in this group [25].

The definition of transient and long-term PoSH varied between individual studies as seen in Table 2. Some definitions only required reduction in serum calcium levels $[6,9$, $12,14,23,24,26]$, while others required reduction in both calcium and PTH levels in defining hypocalcaemia $[17,18]$. Corrected serum calcium levels were generally used but one study used ionised calcium levels [29]. The duration for the definition of long-term PoSH was specified as 6 months in some studies [8, 14] and 12 months in others [6, 27]. Some studies reported rates of transient and long-term hypocalcaemia without providing a definition in the manuscript [11, 13, 15, 16, 19-21].

The British Association of Thyroid and Endocrine surgeons (BAETS) defined transient post-thyroidectomy hypocalcaemia as adjusted serum calcium $<2.10 \mathrm{mmol} / \mathrm{L}$ on day 1 postoperatively [30]. Although this cut-off could be used to standardise the definition of hypoparathyroidism postlaryngectomy, patients have longer inpatient stay following laryngectomy and limiting the definition to day 1 would underestimate the rates of transient hypoparathyroidism. An alternative standard definition could be the lower limit of postoperative PTH levels which have been shown to be a reliable indicator of transient PoSH [31] and predictor of long-term PoSH [32].

To define long-term hypocalcaemia and hypoparathyroidism, the need for calcium/and or vitamin D supplement to maintain normocalcaemia is recommended by the BAETS 
[30]. The European Society of Endocrinology Clinical Guideline also used 6-month duration to diagnose long-term hypoparathyroidism; however, their definition includes a low PTH level [33]. Standardising definitions would enable a fair comparison of $\mathrm{PoSH}$ rates across units and in the literature. In thyroid surgery, the rates of postoperative hypocalcaemia have been shown to vary between 0 and $46 \%$ in a cohort depending on the definition used; highlighting the important of standardised definitions [34].

The risk factors for transient hypocalcaemia identified in this review (total thyroidectomy [6], bilateral neck dissection [9], salvage total laryngectomy [9], total pharyngectomy [10], free jejunum flap [10], and gastric transposition [10]) reflect the extent of surgery involved. Risk factors for PoSH in thyroid surgery such as inadvertent parathyroid gland excision and intraoperative identification of fewer parathyroid glands have not been studied in patients undergoing laryngectomy.

Only one study reporting on preventative measures demonstrated that routine autotransplantation of parathyroid glands may prevent long-term PoSH in patients who had en bloc resection of thyroid and parathyroid glands with total laryngectomy [8]. Parathyroid gland autotransplantation is well reported in thyroid surgery. Other preventative measures evaluated in thyroid surgery include routine peri-operative calcium and vitamin $\mathrm{D}$ supplementation, intraoperative parathyroid identification and use of haemostatic devices (including harmonic scalpel, ligasure) [35]. As in thyroid surgery, preoperative identification and treatment of vitamin $D$ deficiency may help reduce the incidence and severity of post laryngectomy hypocalcaemia. Furthermore, the use of fluorescent imaging [36] and electric impedance spectroscopy [37] has been studied to aid intraoperative parathyroid localisation. These novel preventative measures may guide preservation and direct autotransplantation.

There were no studies on management strategies or treatment protocols for PoSH following laryngectomy. Management of hypocalcaemia following laryngectomy is challenging in the early postoperative phase, particularly in patients with gastric pull up reconstruction. These patients appear to require higher doses of calcium supplementation to normalise serum calcium [22]. In addition, careful monitoring of serum calcium is required to prevent iatrogenic hypercalcaemia [38]. As reported in the literature on thyroid surgery [39, 40], implementation of standard protocols on the identification and early treatment of PoSH after laryngectomy may reduce short-term morbidity, reduce hospital stay and the need for intravenous calcium supplementation.

The systematic review is limited by the sparsity of available data in low to moderate quality case series. There was variability in extent of surgical intervention and the definitions used for transient and long-term PoSH; which precluded meta-analysis.
Further well-designed cohort studies are required to further characterise hypocalcaemia following laryngectomy as good quality data on his complication are lacking. The association between inadvertent parathyroid excision and hypocalcaemia in this group has not been explored. Further work is also needed to identify and evaluate preventative measures. In addition, studies on treatment protocols to guide management are also required.

\section{Conclusion}

Hypocalcaemia following laryngectomy has been reported in a significant proportion of patients. There are limited data on preventative measures and treatment strategies for postlaryngectomy hypoparathyroidism.

Author contributions Conception for this review was by OE and SB. $\mathrm{OE}$ and LS performed literature search and data analysis. OE drafted the manuscript. SB and NB critically reviewed/revised the manuscript.

Funding None.

\section{Compliance with ethical standards}

Conflict of interest The authors declare that they have no conflict of interest.

Open Access This article is licensed under a Creative Commons Attribution 4.0 International License, which permits use, sharing, adaptation, distribution and reproduction in any medium or format, as long as you give appropriate credit to the original author(s) and the source, provide a link to the Creative Commons licence, and indicate if changes were made. The images or other third party material in this article are included in the article's Creative Commons licence, unless indicated otherwise in a credit line to the material. If material is not included in the article's Creative Commons licence and your intended use is not permitted by statutory regulation or exceeds the permitted use, you will need to obtain permission directly from the copyright holder. To view a copy of this licence, visit http://creativecommons.org/licenses/by/4.0/.

\section{References}

1. Edafe O, Antakia R, Laskar N, Uttley L, Balasubramanian SP (2014) Systematic review and meta-analysis of predictors of postthyroidectomy hypocalcaemia. Br J Surg 101(4):307-320

2. Edafe O, Balasubramanian SP (2017) Incidence, prevalence and risk factors for post-surgical hypocalcaemia and hypoparathyroidism. Gland Surg 6(Suppl 1):S59-s68

3. Hannan FM, Thakker RV (2013) Investigating hypocalcaemia. BMJ 346:f2213

4. Underbjerg L, Sikjaer T, Mosekilde L, Rejnmark L (2013) Cardiovascular and renal complications to postsurgical hypoparathyroidism: a Danish nationwide controlled historic follow-up study. J Bone Miner Res 28(11):2277-2285 
5. Underbjerg L, Sikjaer T, Mosekilde L, Rejnmark L (2014) Postsurgical hypoparathyroidism-risk of fractures, psychiatric diseases, cancer, cataract, and infections. J Bone Miner Res 29(11):2504-2510

6. Harris AS, Prades E, Passant CD, Ingrams DR (2018) Hypocalcaemia following laryngectomy: prevalence and risk factors. $\mathrm{J}$ Laryngol Otol 132(11):969-973

7. Murad MH, Sultan S, Haffar S, Bazerbachi F (2018) Methodological quality and synthesis of case series and case reports. BMJ Evid Based Med 23(2):60-63

8. Abd Elmaksoud AEM, Farahat IG, Kamel MM (2015) Parathyroid gland autotransplantation after total thyroidectomy in surgical management of hypopharyngeal and laryngeal carcinomas: a case series. Ann Med Surg 4(2):85-88

9. Basheeth N, O'Cathain E, O'Leary G, Sheahan P (2014) Hypocalcemia after total laryngectomy: incidence and risk factors. Laryngoscope 124(5):1128-1133

10. Clark JR, Gilbert R, Irish J, Brown D, Neligan P, Gullane PJ (2006) Morbidity after flap reconstruction of hypopharyngeal defects. Laryngoscope 116(2):173-181

11. Gurbuz MK, Acikalin M, Tasar S et al (2014) Clinical effectiveness of thyroidectomy on the management of locally advanced laryngeal cancer. Auris Nasus Larynx 41(1):69-75

12. Harrison DF (1979) Surgical management of hypopharyngeal cancer. particular reference to the gastric "pull-up" operation. Arch Otolaryngol. 105(3):149-152

13. Leong SC, Kartha SS, Kathan C, Sharp J, Mortimore S (2012) Outcomes following total laryngectomy for squamous cell carcinoma: one centre experience. Eur Ann Otorhinolaryngol Head Neck Dis 129(6):302-307

14. Lo Galbo AM, de Bree R, Kuik DJ, Lips P, Leemans CR (2010) Paratracheal lymph node dissection does not negatively affect thyroid dysfunction in patients undergoing laryngectomy. Eur Arch Otorhinolaryngol 267(5):807-810

15. Marion Y, Lebreton G, Brevart C, Sarcher T, Alves A, Babin E (2016) Gastric pull-up reconstruction after treatment for advanced hypopharyngeal and cervical esophageal cancer. Eur Ann Otorhinolaryngol Head Neck Dis 133(6):397-400

16. Mortimore S (1998) Hypoparathyroidism after the treatment of laryngopharyngeal carcinoma. J Laryngol Otol 112(11):1058-1060

17. Negm H, Mosleh M, Fathy H, Awad A (2016) Thyroid and parathyroid dysfunction after total laryngectomy in patients with laryngeal carcinoma. Eur Arch Otorhinolaryngol 273(10):3237-3241

18. Okano W, Hayashi R, Omori K, Shinozaki T (2016) Management of the thyroid gland by salvage surgery for hypopharyngeal and cervical esophageal carcinoma after chemoradiotherapy. Jpn J Clin Oncol 46(7):631-634

19. Oosthuizen JC, Leonard DS, Kinsella JB (2012) The role of pectoralis major myofascial flap in salvage laryngectomy: a single surgeon experience. Acta Otolaryngol 132(9):1002-1005

20. Shenson JA, Craig JN, Rohde SL (2017) Effect of preoperative counseling on hospital length of stay and readmissions after total laryngectomy. Otolaryngol Head Neck Surg 156(2):289-298

21. Smith KS, Quiney RE (1987) Endocrine complications of laryngeal and pharyngeal cancer. J Laryngol Otol 101(3):276-282

22. Krespi YP, Wurster CF, Wang TD, Stone DM (1985) Hypoparathyroidism following total laryngopharyngectomy and gastric pull-up. Laryngoscope 95(10):1184-1187

23. Thorp MA, Levitt NS, Mortimore S, Isaacs S (1999) Parathyroid and thyroid function five years after treatment of laryngeal and hypopharyngeal carcinoma. Clin Otolaryngol Allied Sci 24(2):104-108
24. Osborn DA, Jones WI (1968) Parathyroid dysfunction following surgery of the pharynx and larynx. Br J Surg 55(4):277-282

25. Martins AS, Tincani AJ (2006) Thyroidectomy and hypoparathyroidism in patients with pharyngoesophageal tumors. Head Neck 28(2):135-141

26. Buchanan G, West TE, Woodhead JS, Lowy C (1975) Hypoparathyroidism following pharyngolaryngo-oesophagectomy. Clin Oncol 1(2):89-96

27. Panda S, Kumar R, Konkimalla A et al (2019) Rationale behind thyroidectomy in total laryngectomy: analysis of endocrine insufficiency and oncological outcomes. Indian J Surg Oncol 10(4):608-613

28. Saito Y, Kawakubo H, Takami H et al (2019) Thyroid and parathyroid functions after pharyngo-laryngo-esophagectomy for cervical esophageal cancer. Ann Surg Oncol 26(11):3711-3717

29. Farlow JL, Birkeland AC, Rosko AJ et al (2019) Elective paratracheal lymph node dissection in salvage laryngectomy. Ann Surg Oncol 26(8):2542-2548

30. Chadwick DR (2017) Hypocalcaemia and permanent hypoparathyroidism after total/bilateral thyroidectomy in the BAETS registry. Gland Surg 6(Suppl 1):S69-74

31. AlQahtani A, Parsyan A, Payne R, Tabah R (2014) Parathyroid hormone levels 1 hour after thyroidectomy: an early predictor of postoperative hypocalcemia. Can J Surg 57(4):237-240

32. Almquist M, Hallgrimsson P, Nordenström E, Bergenfelz A (2014) Prediction of permanent hypoparathyroidism after total thyroidectomy. World J Surg 38(10):2613-2620

33. Bollerslev J, Rejnmark L, Marcocci C et al (2015) European society of endocrinology clinical guideline: treatment of chronic hypoparathyroidism in adults. Eur J Endocrinol 173(2):G1-20

34. Mehanna HM, Jain A, Randeva H, Watkinson J, Shaha A (2010) Postoperative hypocalcemia - the difference a definition makes. Head Neck 32(3):279-283

35. Antakia R, Edafe O, Uttley L, Balasubramanian SP (2015) Effectiveness of preventative and other surgical measures on hypocalcemia following bilateral thyroid surgery: a systematic review and meta-analysis. Thyroid 25(1):95-106

36. Hillary SL, Guillermet S, Brown NJ, Balasubramanian SP (2018) Use of methylene blue and near-infrared fluorescence in thyroid and parathyroid surgery. Langenbecks Arch Surg 403(1):111-118

37. Hillary SL, Brown BH, Brown NJ, Balasubramanian SP (2020) Use of electrical impedance spectroscopy for intraoperative tissue differentiation during thyroid and parathyroid surgery. World $\mathbf{J}$ Surg 44(2):479-485

38. Leng C, Charlesworth G, Nofal E, P BS (2015) Hypercalcemia following alfacalcidol for post-surgical hypoparathyroidism-an underestimated complication? J Endocrinol Diabetes. 2(5):1-5

39. Stedman T, Chew P, Truran P, Lim CB, Balasubramanian SP (2018) Modification, validation and implementation of a protocol for post-thyroidectomy hypocalcaemia. Ann R Coll Surg Engl 100(2):135-139

40. Wiseman JE, Mossanen M, Ituarte PH, Bath JM, Yeh MW (2010) An algorithm informed by the parathyroid hormone level reduces hypocalcemic complications of thyroidectomy. World J Surg 34(3):532-537

Publisher's Note Springer Nature remains neutral with regard to jurisdictional claims in published maps and institutional affiliations. 